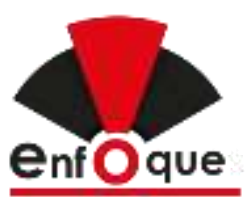

\section{MEJORA CONTINUA EN LAS ORGANIZACIONES A PARTIR DE LA SATISFACCIÓN DE LOS STAKEHOLDERS INTERNOS}

\author{
CONTINUOUS IMPROVEMENT IN ORGANIZATIONS FROM THE \\ SATISFACTION OF INTERNAL STAKEHOLDERS
}

\author{
MELHORIA CONTÍNUA NAS ORGANIZAÇÕES A PARTIR DA SATISFAÇÃO DOS \\ STAKEHOLDERS INTERNOS
}

\section{Erazo Calvopiña Rina Monserrat Nelly Germania Salguero Barba}

Artículo recibido 3 de febrero 2021 | Arbitrado 24 de febrero 2021 | Publicado 01 de abril 2021

\section{Resumen}

La investigación tuvo como objetivo diseñar una propuesta para la mejora continua de la Universidad Técnica de Cotopaxi con base en la satisfacción de los Stakeholders internos que aporte a la retención estudiantil. El enfoque de la investigación fue mixto. Tipo longitudinal, no experimental. Se usó el método inductivo. La población fue establecida por la matricula del período (19-20), el cual estuvo conformada por 9953 estudiantes. El tipo de muestreo, fue de 622 de estudiantes. Sin embargo, para la validación del instrumento se usó el 10\% de la muestra. La técnica de investigación utilizada fue la encuesta y como instrumento se empleó un cuestionario. Como resultado se obtuvo un alto \% de insatisfacción en los factores de espacios para quejas y sugerencias; en trámite de matrículas y en atención del personal administrativo. Por lo que se concluye que es preciso que la Universidad desarrolle un Sistema de Gestión de Organizaciones Educativas.

Palabras clave: Stakeholders; gestión de calidad; mejora continua, organizaciones

\begin{abstract}
The objective of the research was to design a proposal for the continuous improvement of the Technical University of Cotopaxi based on the satisfaction of internal Stakeholders that contributes to student retention. The research focus was mixed. Longitudinal type, not experimental. The inductive method was used. The population was established by enrollment for the period (19-20), which was made up of 9953 students. The type of sampling was 622 students. However, for the validation of the instrument, $10 \%$ of the sample was used. The research technique used was the survey and a questionnaire was used as an instrument. As a result, a high\% of dissatisfaction was obtained in the factors of spaces for complaints and suggestions; in the process of registration and in the attention of the administrative staff. Therefore, it is concluded that the University must develop a Management System for Educational Organizations.
\end{abstract}

Key words: Stakeholders; quality management; continuous improvement, organizations
Erazo Calvopiña Rina Monserrat rinaerazo@gmail.com Orcid: 0000-0002-0982-6429

Universidad Técnica de Cotopaxi, Latacunga, Ecuador.

Master en Administración de Empresas, Magister en Gestión del Turismo. Diplomado en Proyectos Sostenibles y Doctorando en Ciencias Administrativas y Gerenciales. Experiencia en planificación estratégica, empresarial, institucional y territorial, proyectos turísticos en general, desarrollo local y docencia. Consultora de la Corporación para el Desarrollo de Actividades Asistenciales Fundación CODESPA. Universidad Técnica de Cotopaxi, Ecuador.

Nelly Germania Salguero Barba nelly.salguero@utc.edu.ec Orcid: 0000-0002-8866-4121

Universidad Estatal de Bolívar, Bolívar, Ecuador.

Licenciada en Administración Ejecutiva. Diploma superior en Didáctica de la Educación Superior Magister en Planeamiento y Administración Educativos. Doctorante en Ciencias Administrativas y Gerenciales-Universidad de Carabobo Venezuela. Universidad Técnica de Cotopaxi-Latacunga, Ecuador. 


\section{Resumo}

O objetivo da pesquisa foi traçar uma proposta de melhoria contínua da Universidade Técnica de Cotopaxi a partir da satisfação dos Stakeholders internos que contribui para a retenção dos alunos. O foco da pesquisa foi misto. Tipo longitudinal, não experimental. O método indutivo foi usado. A população foi estabelecida pela matrícula do período (19-20), que foi composta por 9.953 alunos. O tipo de amostragem foi de 622 alunos. Porém, para a validação do instrumento, utilizou-se $10 \%$ da amostra. A técnica de pesquisa utilizada foi o survey e um questionário foi utilizado como instrumento. Como resultado, obteve-se alto\% de insatisfação nos fatores de espaços para reclamações e sugestões; no processo de inscrição e no atendimento do pessoal administrativo. Portanto, conclui-se que a Universidade deve desenvolver um Sistema de Gestão das Organizações Educacionais

Palavras chave: Stakeholders; gestão de qualidade; melhoria contínua, organizações

\section{INTRODUCCIÓN}

En América Latina, según estudios del Banco Mundial realizados en 2018, el 42\% de estudiantes universitarios desertan de sus carreras profesionales. Ecuador se encuentra en el tercer lugar con un 32\% de deserción universitaria.

Frente a este fenómeno muchas instituciones de educación superior han realizado estudios sobre los factores de la deserción universitaria (insatisfacción de los Stakeholders internos -estudiantes-) y han buscado diferentes estrategias para la retención estudiantil en sus instituciones; por ejemplo, la Universidad Autónoma de Tamaulipas-México que se ha mantenido en el camino hacia una educación de calidad, estudió los factores asociados a la permanencia de los estudiantes de la licenciatura de enfermería en la UAMM-UAT, los resultados, mostraron que las asociaciones directas se presentaron en los factores como: la actitud y comportamiento, el compromiso, y las condiciones socioeconómicas.

A su vez, en Colombia, la Universidad de la Sabana aplica diferentes programas para lograr la retención estudiantil. Pineda y Peraza (2018) identificaron cuatro categorías:
a) confrontación con la realidad;
b) se preocupan de uno en todo: interés, apoyo y fomento de todas las dimensiones que conforman la vida de un estudiante; c) fortalecimiento académico social de estudiante $y$ su efecto extendido; d) incertidumbre y temor. (p.11).

Por consiguiente, se identifica el éxito de los programas de retención estudiantil, especialmente en aquellos que posibilitaron transformaciones al interior del individuo $y$ además fortalecieron las posibilidades de integrarse en la universidad en el ámbito académico y social.

Se planteó como problema de investigación que el desconocimiento de los factores que afectan la satisfacción de los Stakeholders internos- estudiantes de la Universidad Técnica de Cotopaxi determina que la institución no oriente sus esfuerzos a satisfacer los requerimientos de este grupo de interés interno para la mejora continua de esta organización. Por lo tanto, la investigación tiene como objetivo general, diseñar una propuesta para la mejora continua de la Universidad Técnica de Cotopaxi con base en la satisfacción de los Stakeholders internos (estudiantes) que aporte a la retención estudiantil. 
La justificación de la investigación se basa en que la Universidad Técnica de Cotopaxi está enfocada a la mejora continua de la educación universitaria a través de procesos de gestión óptimos y exitosos dirigidos a sus Stakeholders internos- estudiantes.

\section{Marco teórico}

\section{Stakeholders}

Para Freeman (1984), es "cualquier grupo o individuo que pueda afectar o se vea afectado por la consecución de los propósitos de la empresa" (p. 497).
Un sistema Stakeholders de relaciones entre la organización y sus partes interesadas con los distintos grupos o actores no sólo interactúan de manera bidireccional con la organización, sino que regularmente interactúan también con las otras partes interesadas en forma de red, con lo que pueden lograr intervenir directamente en el funcionamiento de la organización con mayor fuerza. (Ferrary, 2005, p. 116).

Para Gaete (2011), un criterio básico para clasificar a los Stakeholders lo ofrece Freeman (2003), al analizar la definición clásica de las partes interesadas acuñada en 1984.

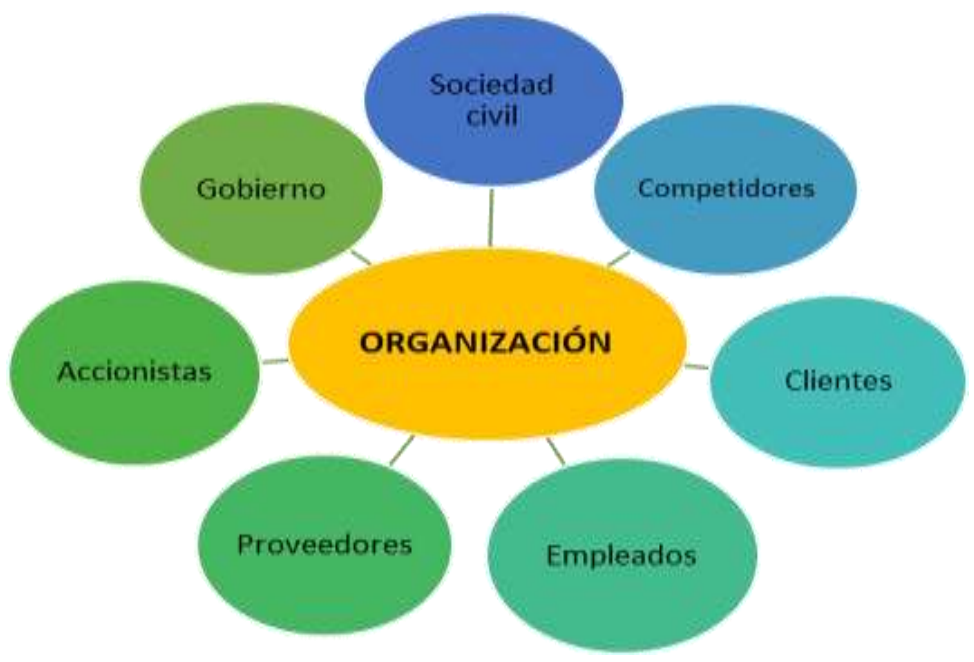

Figura 1. Clasificación de Stakeholders según Freeman, 1984

Es posible distinguir la acepción en un sentido amplio del término que incluye: proveedores, clientes, accionistas, empleados, comunidades, grupos políticos, gobiernos, medios de comunicación, entre otros. En un sentido más estricto, según Freeman (2003) presenta cinco elementos internos: accionistas, clientes, proveedores, empleados y comunidad, eliminando la competencia. A su vez, en ese mismo año, presentó seis grupos de interés externos: el gobierno, los ambientalistas, las organizaciones no gubernamentales, los críticos, los medios de comunicación y otros, sin vincular las flechas. 


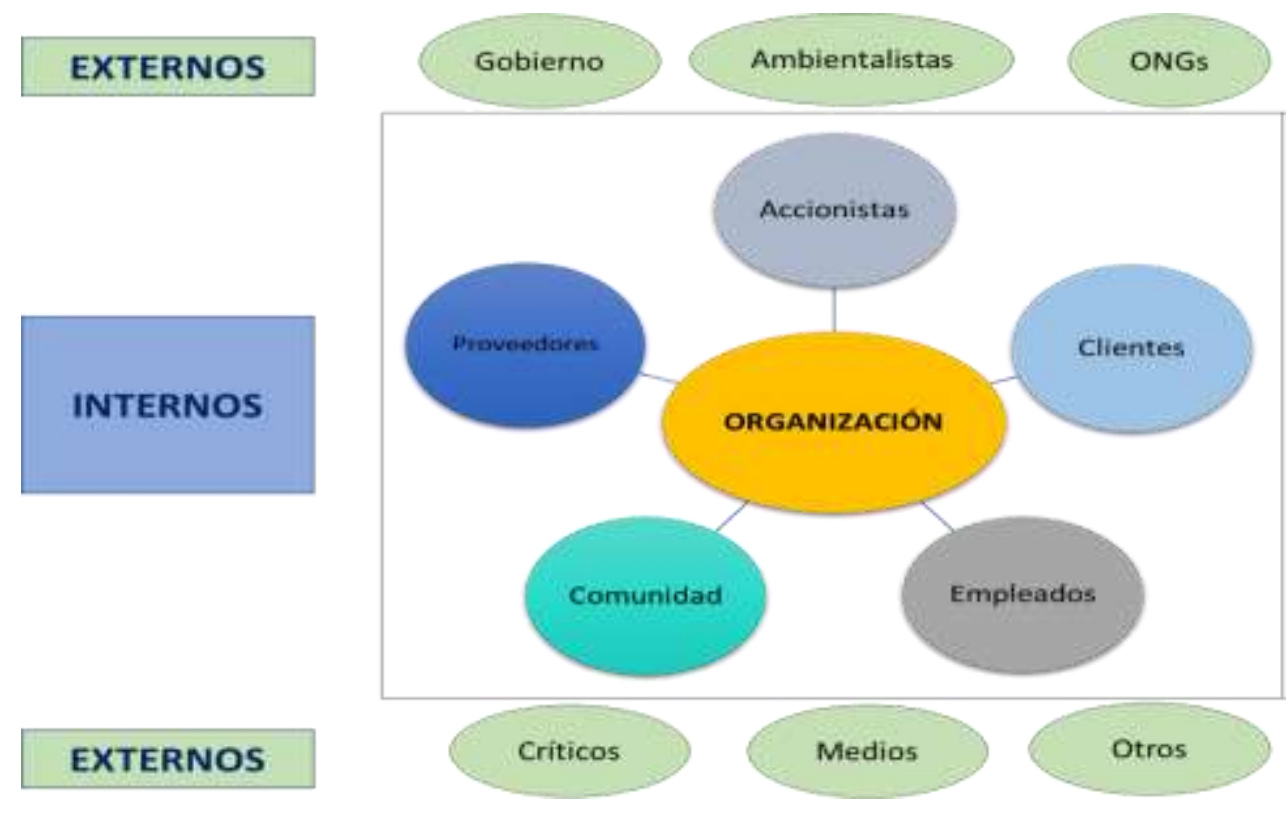

Figura 2. Clasificación actualizada de Stakeholders según Freeman, 2003

Estas figuras diferencian el cambio de perspectiva de los Stakeholders y se ubican por cercanía a la organización, como un mapeo de actores de una organización.

Por otro lado, Kaler (2002), define el término Stakeholder como a un individuo o grupo que hace parte de una organización o tiene una apuesta o interés en ella. En general, se pueden resumir sus definiciones en dos puntos: una desde el punto de vista de derechos adquiridos y otra desde el punto de vista de influenciadores.

Reavill (como se citó en Duque, 2009) identifica que para el caso de la universidad se debe incluir de la siguiente manera:

a) Los estudiantes, como beneficiarios directos del proceso de transformación y como elemento del sistema de interés.

b) Los empleadores, como beneficiarios indirectos del proceso de transformación. Necesita personal entrenado y está dispuesto a pagar por él. Es el comprador si fuera un producto o el cliente si fuera un servicio y es un elemento del sistema de interés ampliado.

c) La familia y dependientes del estudiante. Los padres cuando es joven o quienes dependen cuando es maduro. Esto por cuanto ellos contribuyen directa $\mathrm{o}$ indirectamente en el costo del proceso y pueden ser beneficiarios a través de la expectativa de un nivel superior del estándar de vida una vez el estudiante se gradúe. También es un elemento del sistema de interés ampliado.

d) Las universidades y sus empleados. Esto es, la universidad como entidad y sus empleados, que viven de su actividad en ella. La universidad es el propietario del sistema y los empleados son actores dentro del mismo, siendo ambos miembros del sistema de interés. 
e) El sector de educación secundaria. Es el proveedor del recurso que llega a la universidad y una parte del sistema de interés ampliado.

f) entre otros.

Esta forma de ver a los Stakeholders de las organizaciones de educación superior permitirá acercarse a los estudios de los modelos de educación y mejora continua en la gestión de la calidad de educación superior. (Duque, 2009, p. 28).

Según Duque (2009), en su enfática importancia de los Stakeholders en las universidades determina que entender la organización de la universidad y estudiar sus relaciones con sus Stakeholders requiere necesariamente entender que una organización como la universidad es especialmente sensible a la red social de contactos externos.

Powell y Smith-Doerr (1994) plantean que las acciones sociales, incluidos los hechos económicos, están inmersas en las redes sociales de relaciones, entendidas como un conjunto de elementos vinculados a través de relaciones sociales entre ellos. Esto necesariamente implica que las acciones económicas están influidas siempre por el contexto social en el cual se encuentran, al punto que las acciones sociales, según la premisa de búsqueda de sostenibilidad, han sido supeditadas a las decisiones económicas.

\section{Gestión calidad}

Ovares (2012), define la administración de la educación como la "ciencia y arte que busca el logro de políticas, objetivos y propósitos educativos mediante el desarrollo de instituciones y programas que fomentan la adquisición de conocimientos, actitudes y valores humanos, sustentados en el currículo institucional, comunitario, regional, nacional y universal" (p. 4).

Con relación a la gestión de la calidad en instituciones de educación superior, Adot (2017), define como "el conjunto de actividades cuya responsabilidad e iniciativa para llevarlas a cabo recae en la propia institución" (p. 7).

En el caso de las instituciones de educación superior la calidad interna dependerá de diferentes elementos. Por un lado, se debe tener en cuenta la concepción que el profesorado, como principal grupo de interés que deberá aplicar el SGIC (Sistema de Gestión Interna de Calidad), tenga de la calidad. Por otro lado, se deberá tener en cuenta cuáles son las relaciones entre los diferentes departamentos y grupos de interés de la institución y cómo y quién gestiona los principales procesos de la organización. (Adot, 2017, p. 7)

Según Adot 2017, identifica como elementos de un SGIC el mapa de procesos que es la representación ordenada de los procesos de una organización según unas determinadas categorías y en el que se muestran las relaciones que existen entre ellos; para la construcción del mapa de procesos es necesario seguir cada uno de los siguientes pasos: (p. 17).

- Identificación y secuencia de los procesos.

- Descripción de cada uno de los procesos.

- Identificación de los indicadores y evidencias para el seguimiento y medición de su funcionamiento $y$ alcance de los resultados previstos.

- Mecanismos para la mejora de los procesos basados en evidencias e indicadores. 
Según, Adot (2017), aunque las categorías en las que se clasifican los procesos pueden variar, la clasificación más común es la que se observa a continuación.

- Procesos estratégicos: son aquellos que están vinculados al ámbito de las responsabilidades de la dirección $y$, principalmente, al largo plazo. Se refieren fundamentalmente a procesos de planificación y otros que se consideren ligados a factores clave o estratégicos.

- Procesos operativos: son aquellos ligados directamente con la prestación del servicio, procesos vinculados a la importación de docencia o desarrollo de la investigación.

- Procesos de apoyo: son los que dan apoyo a los procesos operativos. Se suelen referir a procesos relacionados con la gestión de los recursos e infraestructuras, gestión de servicios necesarios directa o indirectamente con la actividad docente, por ejemplo, actualización y mantenimiento de los recursos bibliográficos, gestión y mantenimiento de los laboratorios, aulario, etc.

La calidad de la educación superior de un país, así como su evaluación y supervisión, son esenciales para su bienestar social y económico y constituyen además un factor determinante de la apreciación del sistema de enseñanza superior a nivel internacional.

Las instituciones deben realizar seguimiento y evaluar periódicamente sus programas para garantizar que logran sus objetivos y responden a las necesidades de los estudiantes y de la sociedad. Dichas evaluaciones deben dar lugar a una mejora continua del programa. Como consecuencia de lo anterior, cualquier medida prevista o adoptada, debe comunicarse a todos los interesados. (Agencia Nacional de Evaluación de la Calidad y Acreditación [ANECA], 2015, p. 32).

\section{Mejora continua}

Según la AENOR (2018) la mejora continua se refiere a que la organización debe mejorar continuamente la conveniencia, adecuación y eficacia de los Sistemas de Gestión para Organizaciones Educativas (SGOE), teniendo en cuenta la investigación relevante y las mejores prácticas. La organización debe considerar los resultados del análisis y la evaluación, y las salidas de la revisión por la dirección, para determinar si hay necesidades u oportunidades que deben considerarse como parte de la mejora continua. La organización debe determinar y seleccionar oportunidades de mejora e implementar cualquier acción necesaria para cumplir con los requisitos del estudiante y otros beneficiarios y mejorar la satisfacción de los estudiantes, otros beneficiarios, personal y otras partes interesadas relevantes, incluidos proveedores externos, esto debe incluir:

- La mejora de los productos y servicios para cumplir con los requisitos, así como para abordar las necesidades y expectativas futuras;

- Corregir, prevenir o reducir los efectos no deseados;

- Mejorar el desempeño y la eficacia del SGOE.

Las mejoras pueden incluir corrección, acción correctiva, mejora continua, cambio repentino, innovación y reorganización (p. 53). 
La mejora continua es una cultura, una forma de ser de las personas y de las organizaciones en donde mejorar es la meta fundamental. Para que una institución sea competitiva no basta con implantar mejoras aisladas o accidentales, sino que necesita ponerla en práctica de manera constante, estratégica y sistemática. En esto está el secreto de la continuidad y el éxito de la mejora para lo que se requiere disciplina y perseverancia.

Según Scharager y Aravena (2010), toda institución con intenciones de desarrollar un modelo de gestión de calidad académica debe impulsar una política de calidad y desarrollar procedimientos operativos estándares, con el fin de asegurar el cumplimiento de los objetivos de sus programas.

Estos se deben estipular explícitamente con el fin de desarrollar una cultura que reconozca la importancia del mejoramiento continuo de la calidad y la gestión de la misma en su trabajo. Para lograr lo anterior, la universidad debe desarrollar y ejecutar una estrategia para la mejora continua de la calidad cuya estrategia, política y procedimientos deben tener un status formal y deben estar disponibles a los actores; asimismo, deben incluir el rol de todos los participantes en el sistema.

Es de suma importancia asegurar que los recursos disponibles para el apoyo del aprendizaje sean adecuados y apropiados para cada programa ofrecido. Se debe disponer de un sistema de información horizontal para la fácil recolección de información relevante para la gestión eficaz de sus programas de estudio y otras actividades. El desarrollo y cálculo de indicadores de gestión es absolutamente relevante para evaluar el desempeño de cada escuela. Para ello, se debe contar con acceso a información clave que permita el cálculo eficaz y eficiente de estos indicadores.

González (2004), menciona que la atención a los modelos de evaluación orientados a la mejora de las instituciones educativas exige un acercamiento previo al concepto de evaluación y su incursión en el proceso evaluativo. Más allá de la identificación de este término con sanción, valoración, eficacia o eficiencia, se entiende por evaluación educativa, un proceso sistemático de recopilación de información respecto del sistema general de actuación educativa, en relación con unos criterios o referencias, para formar juicios de valor acerca de una determinada situación y tomar decisiones. El conocimiento de esta realidad incluye, según López Mojarro (1999: 22), los hechos, los procesos que los han generado y los factores que los han condicionado. Estos juicios, tal y como señala Casanova (1992: 31), se utilizarán en la toma de decisiones consecuente con objeto de mejorar la actividad educativa valorada. Se trata, por lo tanto, de realizar una descripción completa de la realidad, aplicando métodos de investigación cuantitativos y cualitativos procedentes de las Ciencias Sociales.

\section{MÉTODO}

El enfoque investigativo de la presente investigación fue Mixto- Inductivo, porque en la recopilación de la información y datos se partió de lo particular a lo general y es mixto porque se realizó una combinación del enfoque cuantitativo y cualitativo. Además, fue de tipo longitudinal porque se comparó datos obtenidos en diferentes oportunidades a la misma población, no experimental porque no se generó ningún ambiente 
controlado. El Método utilizado fue Inductivo porque se analizaron casos particulares, cuyos resultados fueron tomados para extraer conclusiones de carácter general.

La población y muestra, fueron establecidas con base en el número de estudiantes matriculados en el período 20192020 de todas las carreras de la Universidad Técnica de Cotopaxi, el cual estuvo conformada por 9953 estudiantes. El tipo de muestreo fue no probabilístico estratificado, teniendo como estratos a las carreras de la Universidad Técnica de Cotopaxi y como unidades de estudio a los estudiantes de 5to, 6 to y 7 mo semestres, conformado por 622 de estudiantes. Sin embargo, para la validación del instrumento se realizó una prueba piloto, la cual fue aplicada al $10 \%$ de la muestra, es decir 62 estudiantes. Con los resultados de la prueba piloto, se aplicó el coeficiente alfa de Cronbach para la identificación de la consistencia interna y fiabilidad del instrumento

La técnica de investigación utilizada fue la encuesta y como instrumento se empleó un cuestionario, el mismo estuvo conformado por de preguntas cerradas que abordaron 61 factores distribuidos en los siguientes aspectos: Gestión Académica, profesores, instalaciones e Infraestructura, servicios Universitarios, aspectos organizacionales y; relaciones personales.

Para la valoración de cada pregunta se utilizó una escala de tipo Likert, con el siguiente esquema:

Tabla 1. Escala de tipo Likert del instrumento de investigación

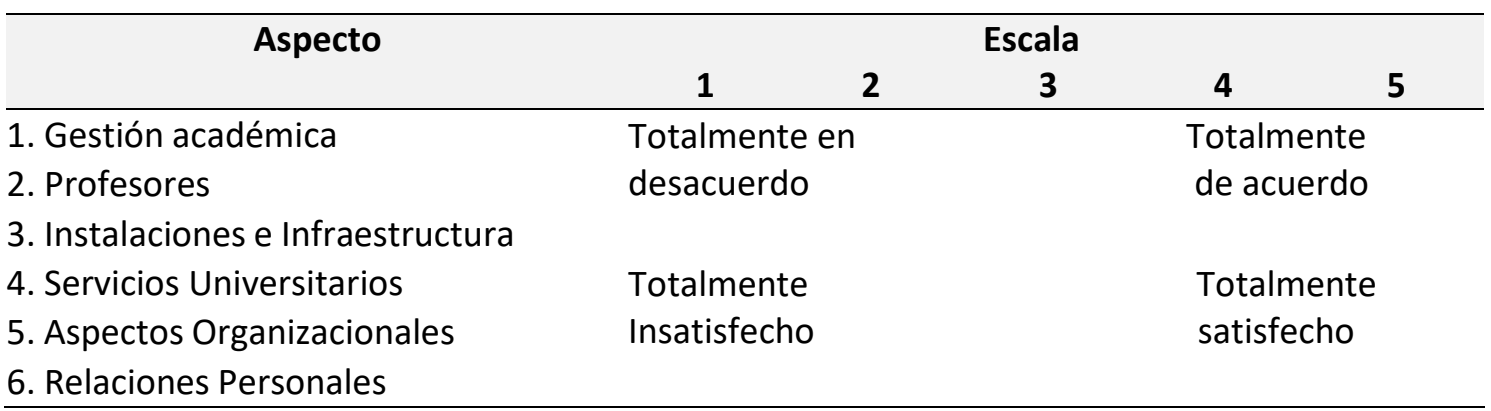

Esta investigación se realizó como la primera toma de datos de un estudio longitudinal que la Universidad Técnica de Cotopaxi tiene planificado realizar en los siguientes años. La tabulación de datos y el análisis estadístico se realizó con el programa SPSS.

\section{RESULTADOS}

A continuación, se presenta el análisis de los resultados enfocados a los 6 aspectos y 61 factores organizados de acuerdo al instrumento investigativo aplicado.

\section{Aspecto 1. Gestión académica}

En la tabla 2 se muestra que, entre los 10 factores de análisis correspondientes al aspecto de Gestión Académica, se identificaron a 3 con más alto nivel de insatisfacción en los estudiantes, el cual están relacionados con el calendario académico y su relación con la planificación del tiempo del estudiante (38,10\%); además las tutorías académicas no son suficientes para que los estudiantes superen las dificultades académicas $(36,66 \%)$ y como tercer factor se presenta que la universidad no organiza 
cursos, talleres y seminarios para que complemente la formación del estudiante $(34,41 \%)$. Los 7 factores restantes presentan un nivel de insatisfacción entre $33,44 \%$ hasta
$21,06 \%$, además, el factor que tiene $38,10 \%$ es uno de los más altos de los 61 factores analizados.

Tabla 2. Satisfacción e insatisfacción, aspecto gestión académica

\begin{tabular}{|c|c|c|c|}
\hline Factor & $\begin{array}{c}\mathbf{( \% )} \\
\text { Insatisfacción }\end{array}$ & $\begin{array}{l}\text { (\%) Mayor } \\
\text { satisfacción }\end{array}$ & Total(\%) \\
\hline $\begin{array}{l}\text { Existe coherencia entre el Plan de estudios y el perfil de } \\
\text { egreso propuesto por la Universidad }\end{array}$ & $32,96 \%$ & $67,04 \%$ & 100,00 \\
\hline $\begin{array}{l}\text { Los contenidos de las asignaturas me ayudan a alcanzar } \\
\text { los resultados de aprendizaje propuestos en cada una } \\
\text { de ellas }\end{array}$ & $30,71 \%$ & $69,29 \%$ & 100,00 \\
\hline $\begin{array}{l}\text { Existe relación entre los contenidos de las asignaturas y } \\
\text { su aplicación en las prácticasPreprofesionales }\end{array}$ & $30,39 \%$ & $69,61 \%$ & 100,00 \\
\hline $\begin{array}{l}\text { El calendario académico me ha permitido planificar mi } \\
\text { tiempo y hacer un buen seguimientode las asignaturas }\end{array}$ & $38,10 \%$ & $61,90 \%$ & 100,00 \\
\hline $\begin{array}{l}\text { La normativa interna que rige las actividades } \\
\text { académicas es suficiente y clara }\end{array}$ & $33,44 \%$ & $66,56 \%$ & 100,00 \\
\hline $\begin{array}{l}\text { Las tutorías académicas son suficientes y me ayudan a } \\
\text { superar las dificultades académicas }\end{array}$ & $36,66 \%$ & $63,34 \%$ & 100,00 \\
\hline $\begin{array}{l}\text { La universidad organiza cursos, talleres y seminarios } \\
\text { para complementar mi formación }\end{array}$ & $34,41 \%$ & $65,59 \%$ & 100,00 \\
\hline $\begin{array}{l}\text { La universidad promueve la participación estudiantil en } \\
\text { proyectos de investigación }\end{array}$ & $25,24 \%$ & $74,76 \%$ & 100,00 \\
\hline $\begin{array}{l}\text { La universidad promueve la participación estudiantil en } \\
\text { proyectos de vinculación }\end{array}$ & $21,06 \%$ & $78,94 \%$ & 100,00 \\
\hline El Plan de estudios es general es bueno & $26,85 \%$ & $73,15 \%$ & 100,00 \\
\hline
\end{tabular}

\section{Aspecto 2. Profesores}

En la tabla 3, se muestra que, entre los 10 factores de análisis correspondientes al aspecto de profesores, se identificaron a 3 con más alto nivel de insatisfacción en los estudiantes, que se refieren a la metodología utilizada por los profesores (37,78\%); en los materiales y recursos didácticos son suficientes para alcanzar los objetivos de la asignatura $(34,41 \%)$ y; lo relacionado con los criterios y procedimientos para la evaluación ayuda al estudiante a su mejoramiento $(29,26 \%)$. 
Tabla 3. Satisfacción e insatisfacción. Aspecto profesores

\begin{tabular}{|c|c|c|c|}
\hline Factor & $\begin{array}{c}\mathbf{( \% )} \\
\text { Insatisfacción } \\
\end{array}$ & $\begin{array}{l}\text { (\%) Mayor } \\
\text { satisfacción }\end{array}$ & Total(\%) \\
\hline Los profesores cumplen con el sílabo de su asignatura & $26,53 \%$ & $73,47 \%$ & 100,00 \\
\hline $\begin{array}{l}\text { La formación de los profesores es adecuada para las } \\
\text { asignaturas que imparten }\end{array}$ & $28,94 \%$ & $71,06 \%$ & 100,00 \\
\hline Los profesores dominan los contenidos de sus asignaturas & $26,85 \%$ & $73,15 \%$ & 100,00 \\
\hline La metodología que utilizan los profesores es adecuada & $37,78 \%$ & $62,22 \%$ & 100,00 \\
\hline $\begin{array}{l}\text { El material didáctico y recursos de aprendizaje que utilizan } \\
\text { los profesores son suficientes para alcanzar los resultados de } \\
\text { aprendizaje en cada asignatura }\end{array}$ & $34,41 \%$ & $65,59 \%$ & 100,00 \\
\hline $\begin{array}{l}\text { Los criterios y procedimientos de evaluación me ayudan a } \\
\text { identificar los aspectos que debo mejorar }\end{array}$ & $29,26 \%$ & $70,74 \%$ & 100,00 \\
\hline Los profesores tienen un trato respetuoso y cordial & $18,01 \%$ & $81,99 \%$ & 100,00 \\
\hline $\begin{array}{l}\text { Los profesores son accesibles y demuestran interés por sus } \\
\text { estudiantes }\end{array}$ & $28,94 \%$ & $71,06 \%$ & 100,00 \\
\hline $\begin{array}{l}\text { Los profesores actúan con ética y la promueven entre los } \\
\text { estudiantes }\end{array}$ & $24,44 \%$ & $75,56 \%$ & 100,00 \\
\hline La actuación de los profesores en general es satisfactoria & $24,28 \%$ & $75,72 \%$ & 100,00 \\
\hline
\end{tabular}

Los 7 factores restantes presentan un nivel de insatisfacción entre $28,94 \%$ hasta $18,01 \%$, además, el factor que tiene $37,78 \%$ es uno de los más altos de los 61 factores analizados.

\section{Aspecto 3. Instalaciones e infraestructura}

Se muestra en la tabla 4, entre los 15 factores de análisis correspondientes al aspecto Instalaciones e Infraestructura, de los cuales se identificaron a los 3 con más alto nivel de insatisfacción en los estudiantes, entre los cuales se destaca el acceso a internet $(41,96 \%)$; las instalaciones en relación a cafeterías (38,75\%) y; lo referente a los baños de la universidad (34,08\%). Los 12 factores restantes presentan un nivel de insatisfacción entre $32,64 \%$ hasta $16,24 \%$. Y el factor que tiene $41,96 \%$ es uno de los más altos de los 61 factores analizados. 
Tabla 4. Satisfacción e insatisfacción, aspecto Instalaciones e Infraestructura

\begin{tabular}{lccc}
\hline \multicolumn{1}{c}{ Factor } & $\begin{array}{c}\text { (\%) De } \\
\text { acuerdo }\end{array}$ & $\begin{array}{c}\text { (\%) } \\
\text { Desacuerdo }\end{array}$ & Total (\%) \\
\hline $\begin{array}{l}\text { Disponibilidad bibliográfica para el estudio (libros } \\
\text { físicos) }\end{array}$ & $28,14 \%$ & $71,86 \%$ & $\mathbf{1 0 0 , 0 0}$ \\
$\begin{array}{l}\text { Disponibilidad bibliográfica para el estudio(libros } \\
\text { digitales) }\end{array}$ & $26,69 \%$ & $73,31 \%$ & $\mathbf{1 0 0 , 0 0}$ \\
Laboratorios y/o talleres & $28,14 \%$ & $71,86 \%$ & $\mathbf{1 0 0 , 0 0}$ \\
Laboratorios de cómputo & $25,72 \%$ & $74,28 \%$ & $\mathbf{1 0 0 , 0 0}$ \\
$\begin{array}{l}\text { Aulas de clase (ventilación, iluminación, pupitres y } \\
\text { sillas) }\end{array}$ & $32,64 \%$ & $67,36 \%$ & $\mathbf{1 0 0 , 0 0}$ \\
$\begin{array}{l}\text { Equipamiento de las aulas de clase (proyector y } \\
\text { computador) }\end{array}$ & $19,45 \%$ & $80,55 \%$ & $\mathbf{1 0 0 , 0 0}$ \\
Auditorios & $32,64 \%$ & $67,36 \%$ & $\mathbf{1 0 0 , 0 0}$ \\
Cafeterías & $38,75 \%$ & $61,25 \%$ & $\mathbf{1 0 0 , 0 0}$ \\
Conectividad (Acceso a internet) & $41,96 \%$ & $58,04 \%$ & $\mathbf{1 0 0 , 0 0}$ \\
Aulas virtuales & $16,24 \%$ & $83,76 \%$ & $\mathbf{1 0 0 , 0 0}$ \\
Baños & $34,08 \%$ & $65,92 \%$ & $\mathbf{1 0 0 , 0 0}$ \\
Áreas verdes & $19,61 \%$ & $80,39 \%$ & $\mathbf{1 0 0 , 0 0}$ \\
Instalaciones deportivas & $21,86 \%$ & $78,14 \%$ & $\mathbf{1 0 0 , 0 0}$ \\
Instalaciones e infraestructura en general & $17,85 \%$ & $82,15 \%$ & $\mathbf{1 0 0 , 0 0}$ \\
\hline
\end{tabular}

\section{Aspecto 4. Servicios universitarios}

En la tabla 5, se aprecia que, de los 15 factores de análisis correspondientes al aspecto Servicios Universitarios, se identificaron a 3 con más alto nivel de insatisfacción en los estudiantes, entre los cuales se destacan, el que no existen canales para realizar sugerencias y quejas $(56,11 \%)$; la asistencia odontológica no es buena
(50,48\%) y; la necesidad de movilidad e intercambios estudiantiles (49,84\%). Además, los 9 factores restantes presentan un nivel de insatisfacción entre $45,66 \%$ hasta 27,49\%. El factor, canales para realizar sugerencias y quejas que, tiene $56,11 \%$ es uno de los más altos de los 61 factores analizados. 
Tabla 5. Satisfacción e insatisfacción, Aspecto Servicios Universitarios

\begin{tabular}{lccc}
\hline \multicolumn{1}{c}{ Factor } & $\begin{array}{c}\text { (\%) de } \\
\text { Acuerdo }\end{array}$ & $\begin{array}{c}\text { (\%) } \\
\text { Desacuerdo }\end{array}$ & $\begin{array}{c}\text { Total } \\
\text { (\%) }\end{array}$ \\
\hline Asistencia odontológica & $50,48 \%$ & $49,52 \%$ & $\mathbf{1 0 0 , 0 0}$ \\
Atención psicológica & $44,37 \%$ & $55,63 \%$ & $\mathbf{1 0 0 , 0 0}$ \\
Becas y ayudas económicas & $41,96 \%$ & $58,04 \%$ & $\mathbf{1 0 0 , 0 0}$ \\
Movilidad e intercambios estudiantiles & $49,84 \%$ & $50,16 \%$ & $\mathbf{1 0 0 , 0 0}$ \\
Orientación profesional y laboral & $40,19 \%$ & $59,81 \%$ & $\mathbf{1 0 0 , 0 0}$ \\
Actividades culturales, deportivas y sociales & $28,14 \%$ & $71,86 \%$ & $\mathbf{1 0 0 , 0 0}$ \\
Canales para realizar sugerencias y quejas & $56,11 \%$ & $43,89 \%$ & $\mathbf{1 0 0 , 0 0}$ \\
Seguridad en el campus universitario & $45,66 \%$ & $54,34 \%$ & $\mathbf{1 0 0 , 0 0}$ \\
Servicio de limpieza & $27,49 \%$ & $72,51 \%$ & $\mathbf{1 0 0 , 0 0}$ \\
Servicio de fotocopias & $35,85 \%$ & $64,15 \%$ & $\mathbf{1 0 0 , 0 0}$ \\
Valoración general de los servicios universitarios & $29,10 \%$ & $70,90 \%$ & $\mathbf{1 0 0 , 0 0}$ \\
\hline
\end{tabular}

\section{Aspecto 5. Aspectos organizacionales}

Se muestra en la tabla 6 que, de los 12 factores de análisis correspondientes al Aspectos Organizacionales, se identificaron a solo 3 con el más alto nivel de insatisfacción en los estudiantes, entre los cuales resalta los factores sobre; no existen espacios para quejas y sugerencias (49,52\%); además existen dificultad en la comunicación interna
$(35,69 \%)$ y; existe insatisfacción en lo que es la atención por parte del personal administrativo (34,73\%). Por otro lado, los 6 factores restantes presentan un nivel de insatisfacción entre $33,44 \%$ hasta $25,40 \%$. Así que, el factor de quejas y sugerencias tiene $49,52 \%$ es uno de los más altos de los 61 factores analizados.

Tabla 6. Satisfacción e insatisfacción, Aspectos Organizacionales

\begin{tabular}{lccc}
\hline \multicolumn{1}{c}{ Factor } & (\%) De acuerdo & $\begin{array}{c}\text { (\%) } \\
\text { Desacuerdo }\end{array}$ & $\begin{array}{c}\text { Tota } \\
\text { I(\%) }\end{array}$ \\
\hline Trámites de matrículas & $33,44 \%$ & $66,56 \%$ & $\mathbf{1 0 0 , 0 0}$ \\
Gestión del expediente académico & $31,67 \%$ & $68,33 \%$ & $\mathbf{1 0 0 , 0 0}$ \\
Atención del personal administrativo & $34,73 \%$ & $65,27 \%$ & $\mathbf{1 0 0 , 0 0}$ \\
Información en la página web & $25,40 \%$ & $74,60 \%$ & $\mathbf{1 0 0 , 0 0}$ \\
Información disponible en redes sociales & $25,72 \%$ & $74,28 \%$ & $\mathbf{1 0 0 , 0 0}$ \\
Espacios para quejas y sugerencias & $49,52 \%$ & $50,48 \%$ & $\mathbf{1 0 0 , 0 0}$ \\
$\begin{array}{l}\text { Estrategias para conocer y compartir la } \\
\text { misión, visión y valores de la universidad }\end{array}$ & $29,42 \%$ & $70,58 \%$ & $\mathbf{1 0 0 , 0 0}$ \\
$\begin{array}{l}\text { Valoración general de los aspectos } \\
\text { organizacionales }\end{array}$ & $30,55 \%$ & & \\
\hline
\end{tabular}




\section{Aspecto 6. Relaciones personales}

El aspecto Relaciones Personales se muestra en la tabla 7 , y tiene 5 factores de análisis, de los cuales se identificaron a 3 con el más alto nivel de insatisfacción en los estudiantes, siendo relevante las relaciones personales con las autoridades $(39,07 \%)$; las relaciones personales con los compañeros/as
$(28,30 \%)$ y; las relaciones personales con los profesores (26,21\%). Los 2 factores restantes presentan un nivel de insatisfacción entre $15,11 \%$ hasta $9,65 \%$. El factor, Relaciones personales con las autoridades, tiene $39,07 \%$ y es uno de los más altos de los 61 factores analizados.

Tabla 7. Satisfacción e insatisfacción, Aspecto Relaciones Personales

\begin{tabular}{lccc}
\hline \multicolumn{1}{c}{ Factor } & $\begin{array}{c}\text { (\%) De } \\
\text { acuerdo }\end{array}$ & $\begin{array}{c}\text { (\%) } \\
\text { Desacuerdo }\end{array}$ & $\begin{array}{c}\text { Total } \\
\text { (\%) }\end{array}$ \\
\hline Relaciones personales con mis profesores & $26,21 \%$ & $73,79 \%$ & $\mathbf{1 0 0 , 0 0}$ \\
Relaciones personales con las autoridades & $39,07 \%$ & $60,93 \%$ & $\mathbf{1 0 0 , 0 0}$ \\
Relaciones personales con mi familia & $9,65 \%$ & $90,35 \%$ & $\mathbf{1 0 0 , 0 0}$ \\
Valoración general de mis relaciones personales & $15,11 \%$ & $84,89 \%$ & $\mathbf{1 0 0 , 0 0}$ \\
\hline
\end{tabular}

Con base a los resultados expuestos, a continuación en la Tabla 8 se destaca un resumen correspondiente a los 3 factores que tuvieron los más altos niveles de insatisfacción por cada uno de los 6 aspectos:

Tabla 8 Resumen de factores con niveles de insatisfacción más altos

\begin{tabular}{lcl}
\hline Aspecto & $\begin{array}{c}\text { (\%) } \\
\text { Insatisfacción }\end{array}$ & \multicolumn{1}{c}{ Factor } \\
\hline $38,10 \%$ & $\begin{array}{l}\text { Calendario académico les ha permitido planificar su tiempo y } \\
\text { hacer un buen seguimiento de las asignaturas }\end{array}$ \\
$\begin{array}{lll}\text { 1. Gestión } \\
\text { Académica }\end{array}$ & $\begin{array}{l}\text { Las tutorías académicas son suficientes y les ayudan a superar } \\
\text { las dificultades académicas }\end{array}$ \\
& $34,41 \%$ & $\begin{array}{l}\text { La universidad organiza cursos, talleres y seminarios para } \\
\text { complementar mi formación }\end{array}$ \\
$37,78 \%$ & $\begin{array}{l}\text { La metodología utilizada por los profesores } \\
\text { El material didáctico y recursos de aprendizaje que utilizan los } \\
\text { profesores son suficientes para alcanzarlos resultados de } \\
\text { aprendizaje en cada asignatura }\end{array}$ \\
$34,41 \%$ & $\begin{array}{l}\text { Los criterios y procedimientos de evaluación me ayudan a } \\
\text { identificar los aspectos que debo mejorar } \\
\text { Conectividad (Acceso a internet) }\end{array}$ \\
\hline $41,96 \%$ &
\end{tabular}




\begin{tabular}{lrl}
\hline Aspecto & \multicolumn{1}{c}{$\begin{array}{c}\text { (\%) } \\
\text { Insatisfacción }\end{array}$} & \multicolumn{1}{c}{ Factor } \\
\hline $\begin{array}{l}\text { 3. Instalaciones e } \\
\text { Infraestructura }\end{array}$ & $38,75 \%$ & Cafeterías \\
& $34,08 \%$ & Baños \\
& $56,11 \%$ & Canales para realizar sugerencias y quejas \\
4. Servicios & $50,48 \%$ & Asistencia odontológica \\
Universitarios & $49,84 \%$ & Movilidad e intercambios estudiantiles \\
& $49,52 \%$ & Espacios para quejas y sugerencias \\
5. Aspectos & $35,69 \%$ & Trámite de matrículas \\
Organizacionales & $34,73 \%$ & Atención del personal administrativo \\
& $39,07 \%$ & Relaciones personales con las autoridades de la Universidad \\
\hline $\begin{array}{l}\text { 6. Relaciones } \\
\text { Personales }\end{array}$ & $28,30 \%$ & Relaciones personales con compañeros/as \\
\hline
\end{tabular}

Posterior al análisis de los resultados, se puede resumir que los aspectos con mayor insatisfacción fueron: Servicios Universitarios con altos porcentajes en sus factores, el $56,11 \%$ en Canales para realizar sugerencias y quejas; el $50,48 \%$ en Asistencia odontológica y; $49,84 \%$ en Movilidad e intercambios estudiantiles. Además, en lo que se refiere a Aspectos Organizacionales también tiene factores con altos porcentajes de insatisfacción, tal es el caso del 49,52\% en Espacios para quejas y sugerencias; en Trámite de matrículas con $35,69 \%$ y en Atención del personal administrativo con $34,73 \%$; de esta manera afecta a la retención estudiantil en la Universidad Técnica de Cotopaxi.
Propuesta para la mejora continua de la Universidad Técnica de Cotopaxi con base en la satisfacción de los Stakeholders internos (estudiantes) que aporte a la retención estudiantil

Después de revisar los instrumentos estratégicos que tiene la Universidad Técnica de Cotopaxi para su desarrollo, se utiliza al Plan Estratégico de Desarrollo Institucional, en el cual, se observa cuatro ejes estratégicos que luego del proceso técnico se concretan en los 4objetivos estratégicos, que son los siguientes: 
1. Formación- "Consolidar la formación integral de los estudiantes en grado y posgrado, con calidad y excelencia académica para que alcancen reconocimiento y liderazgo a nivel nacional."

2. Investigación. "Incrementar la producción científica y tecnológica para generar conocimiento que contribuya a mejorar las condiciones de vida de la sociedad."

3. Vinculación. "Fortalecer la vinculación entre la universidad y la sociedad para alcanzar el desarrollo social, comunitario y productivo de la región y del país, a través de alianzas nacionales e internacionales."

4. Gestión. "Incrementar la eficiencia y eficacia de la gestión institucional."

Posterior, con base a los 3 factores con mayor porcentaje de insatisfacción de los 6 aspectos, se plantean estrategias para mejorar la satisfacción del estudiante y lograr la retención estudiantil; por cada estrategia se desglosan las principales actividades, el conjunto de éstas últimas conforman los diferentes posibles planes y proyectos que ejecutarán las diferentes Direcciones de la Universidad Técnica de Cotopaxi, de acuerdo a su organigrama estructural y sus competencias. Todos estos elementos se consolidan en una Matriz de Estrategias para la mejora continua.

\section{Premisas para su implementación}

Las premisas para la implementación de la presente propuesta de mejora continua son: la ejecución y evaluación de las estrategias con sus planes y proyectos para la mejora continua de la Universidad Técnica de Cotopaxi, específicamente en los factores de mayor insatisfacción de los Stakeholders internos (estudiantes) con enfoque a la retención estudiantil.

Para la implementación de la propuesta, se debe proporcionar la importancia necesaria por parte de las Direcciones responsables de cada estrategia $y$; especialmente la Dirección de Planeamiento y Desarrollo Institucional tiene un papel fundamental, ya que debe ser la encargada de la supervisión de la ejecución y posteriormente de la evaluación de los resultados alcanzados; es importante recalcar que la Dirección de Evaluación y Aseguramiento de la Calidad tiene una participación importante en la evaluación de los resultados para la mejora continua $y$; también se debe tomar en cuenta la participación activa de toda la comunidad universitaria, ya que será de gran ayuda para alcanzar los resultados propuestos. a continuación se presenta el modelo para la implementación de la propuesta. 


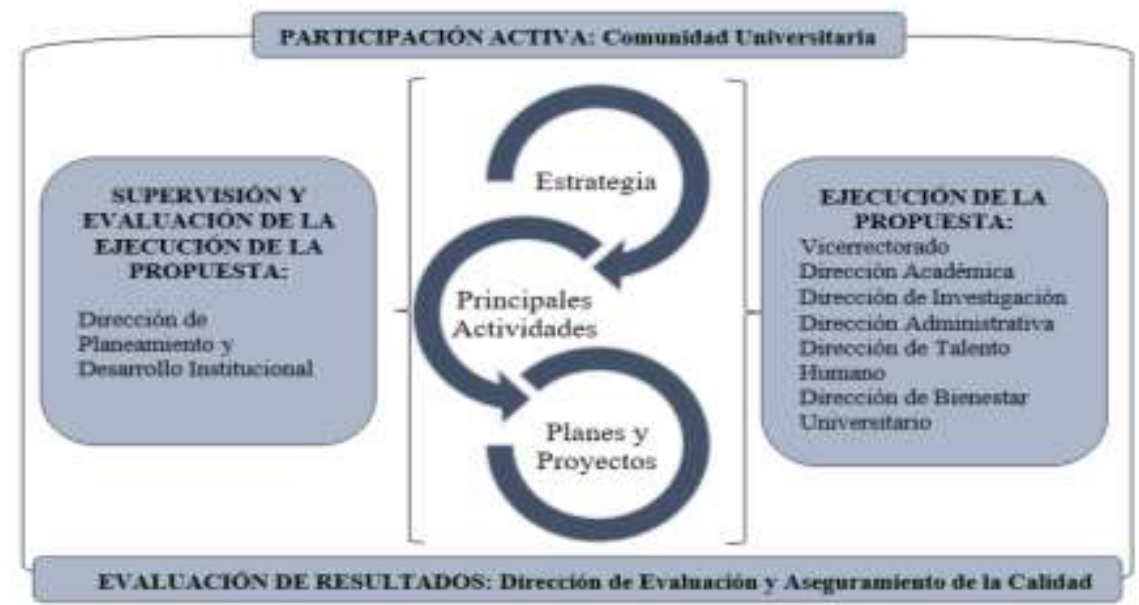

Figura 3. Modelo para la implementación de la propuesta

Validación de la propuesta para la mejora continua de la Universidad Técnica de Cotopaxi con base en la satisfacción de los Stakeholders internos (estudiantes) que aporte a la retención estudiantil

En el proceso de evaluación de expertos de la propuesta para la mejora continua de la Universidad Técnica de Cotopaxi, se aplicó una entrevista de tipo semiestructurada, es decir, que alternando preguntas estructuradas con preguntas espontáneas se logró obtener los resultados deseados.

Se seleccionó como experto a un docente de la Universidad Técnica de Cotopaxi que tiene asignada su carga horaria de Gestión a la Dirección de Evaluación y Aseguramiento de la Calidad por varios períodos académicos y que conoce de cerca los procesos que se realizan en la universidad, tanto desde el punto de vista del aseguramiento de la calidad como de la planificación y gestión institucional.

Al experto se les dio a conocer la contextualización de la investigación realizada, es decir, se le informó que entre los meses de noviembre y diciembre del 2019 se realizó un estudio sobre el nivel de satisfacción de los Stakeholders internos (estudiantes) de la Universidad, para la identificación de los 61 factores determinantes en la satisfacción de los mismos comprendidos en 6 aspectos: Gestión académica, profesores, instalaciones e Infraestructura, servicios Universitarios, aspectos organizacionales y relaciones personales.

Este estudio arrojó información muy importante que será de gran ayuda para la mejora continua institucional. Se dio mayor importancia a los 3 factores por cada aspecto que tienen mayor grado de insatisfacción en los estudiantes. Por ello, se desarrolló una propuesta de mejora continua enfocado a la satisfacción de los Stakeholders internos (estudiantes), las estrategias son las siguientes:

- Reformulación de horarios académicos estudiantiles.

- Creación de un sistema para las tutorías académicas. 
- Programación anual de cursos, talleres y seminarios complementarios a la formación académica.

- Actualización de las capacidades docentes en la implementación de metodologías, recursos de aprendizaje y evaluación de educación superior.

- Repotenciación de la conectividad con tecnología de punta.

- Mejoramiento de los espacios públicos para los estudiantes

- Implementación de un sistema de sugerencias y quejas.

- Repotenciación del área odontológica.

- Promoción del intercambio estudiantil

- Transversalización de la participación de los estudiantes en procesos de toma de decisiones y de gestión universitaria.

- Optimización de los procesos de matrículas.

- Fortalecimiento de las competencias del personal administrativo con calidad humana y atención al estudiante

- Creación de espacios de esparcimiento, de intercambio y de convivencia entre todos los Stakeholders internos.

Luego, se realizó una introducción a la entrevista en el marco de la gestión, evaluación y aseguramiento de la calidad institucional, solicitando el criterio del experto y su validación a la propuesta para la mejora continua de la Universidad Técnica de Cotopaxi; la misma, es un conjunto de estrategias desagregada en principales actividades; alineadas a los objetivos estratégicos institucionales comprendidos en el Plan de Desarrollo Institucional (PEDI 20162020); además la propuesta integra posibles planes y proyectos que ejecutarán las diferentes Direcciones de la Universidad
Técnica de Cotopaxi, según su competencia identificada en el organigrama estructural institucional.

\section{Evaluación de resultados}

Los resultados obtenidos en las entrevistas realizadas, tanto al experto como a los estudiantes fueron de gran importancia para la validación de la presente propuesta, en concordancia con lo expuesto por ellos, se identifica que existe la aceptación y validación de la presente propuesta.

Los entrevistados en general aceptaron las estrategias propuestas y la aplicación. Ya que, los estudiantes se sintieron motivados en que se pueda aplicar y que la mejora continua sea una realidad, especialmente en los factores identificados con mayor insatisfacción en los estudiantes.

El experto aprobó la presente propuesta, con las consideraciones necesarias en relación a la aceptación de las autoridades institucionales, específicamente refiriéndose al presupuesto y a la realidad de la Universidad, sin embargo, las estrategias propuestas tienen la orientación técnica necesaria para ser aplicadas e incorporadas en el nuevo Plan Estratégico de Desarrollo Institucional y; de igual manera, con un proceso de coordinación con la Dirección de Planeamiento para la incorporación de las estrategias y sobre todo para que cada Dirección se encargue de la ejecución.

Finalmente, es importante mencionar que la aplicación de la presente propuesta se encuentra en manos de las autoridades de la Universidad, y que los organismos técnicos como la Dirección de Evaluación y Aseguramiento de la Calidad de la Educación y la Dirección de Planeamiento podrían articular con las demás direcciones para su 
implementación y para la incorporación de estas estrategias en el nuevo Plan Estratégico de Desarrollo Institucional.

\section{Resultados esperados de la propuesta}

Los resultados esperados de la presente propuesta es el aumento en la satisfacción del estudiante en su bienestar universitario, el incremento del rendimiento académico de los estudiantes, el alza en el índice de participación estudiantil en la toma de decisiones y de gestión universitaria y finalmente; lograr la mejora continua en la Universidad Técnica de Cotopaxi, con base en la satisfacción de sus Stakeholders internos (estudiantes). A continuación, los resultados esperados por cada una de las estrategias propuestas:

Tabla 9 Resultados esperados de la Propuesta

\begin{tabular}{cl}
\hline $\mathbf{N}^{\circ}$ & \multicolumn{1}{c}{ Estrategia } \\
\hline $\mathbf{1}$ & $\begin{array}{l}\text { Reformulación de horarios académicos } \\
\text { estudiantiles. }\end{array}$ \\
$\mathbf{2}$ & $\begin{array}{l}\text { Creación de un sistema para las tutorías } \\
\text { académicas. }\end{array}$
\end{tabular}

3 Programación anual de cursos, talleres y seminarios complementarios a la formación académica.

4 Actualización de las capacidades docentes en la implementación de metodologías, recursos de aprendizaje y evaluación de educación superior.

5 Repotenciación de la conectividad con tecnología de punta.

$6 \quad$ Mejoramiento de los espacios públicos para los estudiantes

7 Implementación de un sistema de sugerencias y quejas.

9 Promoción del intercambio estudiantil

10 Transversalización de la participación de los estudiantes en procesos de toma de decisiones y de gestión universitaria.

\section{Resultado}

Optimización del tiempo de los estudiantes para su desarrollo académico.

Estudiantes con mejor rendimiento académico con base en el soporte de las tutorías académicas.

Alto índice de estudiantes que participan en talleres y seminarios complementarios a la formación académica.

Estudiantes con mejor rendimiento académico con base en la implementación de metodologías, recursos de aprendizaje y evaluación de educación superior por parte de los docentes.

Incremento del rendimiento académico con base en la entrega puntual de tareas on line y participación en las plataformas virtuales.

Incremento en la satisfacción del estudiante con relación a su estadía en la Universidad.

Reducción de quejas no atendidas.

Alto índice de estudiantes que participan en intercambios estudiantiles.

Alto índice de participación estudiantil en la toma de decisiones y de gestión universitaria. 


\begin{tabular}{lll}
\hline $\mathbf{N}^{\circ}$ & \multicolumn{1}{c}{ Estrategia } & \multicolumn{1}{c}{ Resultado } \\
\hline 11 & $\begin{array}{l}\text { Optimización en los procesos de } \\
\text { matrículas. }\end{array}$ & $\begin{array}{l}\text { Optimización de los recursos institucionales y } \\
\text { estudiantiles en los procesos de matrícula. }\end{array}$ \\
$\mathbf{1 2} \begin{array}{l}\text { Fortalecimiento de las competencias del } \\
\text { personal administrativo con calidad } \\
\text { humana y atención al estudiante }\end{array}$ & $\begin{array}{l}\text { Incremento en la satisfacción del estudiante en } \\
\text { su estadía y gestión en la Universidad. }\end{array}$ \\
$\begin{array}{l}\text { Creación de espacios de esparcimiento, } \\
\text { de intercambio y de convivencia entre } \\
\text { todos los Stakeholders internos. }\end{array}$ & $\begin{array}{l}\text { Incremento en la satisfacción del estudiante en } \\
\text { su bienestar universitario. }\end{array}$ \\
\end{tabular}

\section{CONCLUSIONES}

Una vez finalizado el desarrollo de la investigación se puede concluir lo siguiente: la satisfacción de los Stakeholders internos (estudiantes) de la Universidad Técnica de Cotopaxi es alta, pero existen factores que tienen un importante porcentaje de insatisfacción, estos se convierten en factores determinantes para el planteamiento de mejora continua orientado a la retención estudiantil.

Además, la propuesta de estrategias para la mejora continua de la Universidad Técnica de Cotopaxi ayuda a corregir las deficiencias que actualmente está provocando insatisfacción en los estudiantes y disminuyendo la retención estudiantil.

De esta manera, la presente propuesta está dirigida específicamente a la mitigación del nivel de insatisfacción de los 3 factores de cada uno de los 6 aspectos estudiados, para esto, es preciso que la Universidad Técnica de Cotopaxi desarrolle un Sistema de Gestión de Organizaciones Educativas (SGOE) como demanda la Norma Española sobre Organizaciones Educativas UE- ISO 21001.

Las estrategias propuestas, incluyendo la alineación a los objetivos estratégicos Institucionales de la Universidad, las principales actividades y los posibles planes y proyectos que derivan de las mencionadas estrategias, fueron validadas por los protagonistas de la investigación, los estudiantes quienes fueron la motivación a la implementación de esta propuesta; de igual manera, el experto en evaluación y aseguramiento de la calidad de la educación valida la propuesta con la perspectiva de que las autoridades aprueben su implementación y financiamiento para el logro de un mejor nivel de retención estudiantil y la mejora continua de la Universidad Técnica de Cotopaxi, Ecuador.

\section{REFERENCIAS}

Adot, E. (2017). Sistema de gestión interna de la calidad. Universidad Oberta de Cataluña, 1(1), 1-48

Agencia Nacional de Evaluación de la Calidad y Acreditación, ANECA (2015). Criterios y directrices para el Aseguramiento de la Calidad en el Espacio Europeo de Educación Superior (ESG). ANECA, 1(1), 134

Asociación Española de Normalización y Certificación. AENOR (2018). Organizaciones educativas

Casanova, A. (1992). La evaluación; garantía de calidad para el centro educativo. España. Editorial Luis Vives.

Duque, E. (2009). La gestión de la universidad como elemento básico del sistema universitario una reflexión desde la 
perspectiva de los Stakeholders. Revista de Ciencias Administrativas y Sociales, 19(1), 25-41

Ferrary M. (2005). A Stakeholder perspective of human resource management. $A$ European perspective. PalgraveMacmillan, 1(1), 104-124

Freeman, E. (1984). Strategic Management: A Stakeholder Approach. Boston: Pitman Publishing Inc. Reimpresión en 2010 por Cambridge University Press.

Freeman, E. (2003). Stakeholder Theory. The Blackwell encyclopedia of management. 2(1), 496-500

Gaete, R. (2011). Identificación de los Stakeholders de las universidades. Revista de Ciencias Sociales, 17(3), 486-499

González, I. (2004). Modelos de evaluación de la calidad orientados a la mejora de las instituciones educativa. Revista de Educación 6 (p. 155-169) Universidad de Huelva

Kaler, J. (2002), Morality and strategy in stakeholder identification. Journal of Business Ethics, 39: 91-99
López, M. (1999). A la calidad por la evaluación. Madrid: Escuela Española

Ovares, E. (2012). Análisis de las estrategias para la prevención de la deserción y retención de la población estudiantil que lleva a cabo el personal del docente $y$ administrativo del Liceo de Miramar, de la Dirección Regional de Educación de Puntarenas, Costa Rica. Revista Gestión de la Educación, 2(2), 1-27

Pineda, E. y Tello, C. (2018). ¿Ciencia, técnica y arte?: análisis crítico sobre algunas posturas del problema del estatus epistemológico de la Administración. Revista Logos Ciencia y Tecnología, 10(4), 112-130

Powell, W., y Smith-Doerr, L. (1994). Networks and economic life. En: Smelser y Swedberg (Eds.). Handbook of Economic Sociology (pp. 368-402). Princeton, NJ: Princeton University Press

Scharager, J., y Aravena, M. (2010). Impacto de las políticas de aseguramiento de la calidad en programas de educación superior un estudio exploratorio. Fundación Dialnet 